\title{
BMO RATIONAL APPROXIMATION AND ONE-DIMENSIONAL HAUSDORFF CONTENT
}

\author{
JOAN VERDERA
}

\begin{abstract}
Let $X \subset \mathbf{C}$ be compact and let $f \in \operatorname{VMO}(\mathbf{C})$. We give necessary and sufficient conditions on $f$ and $X$ for $f_{\mid X}$ to be the limit of a sequence of rational functions without poles on $X$ in the norm of $\operatorname{BMO}(X)$, the space of functions of bounded mean oscillation on $X$. We also characterize those compact $X \subset \mathbf{C}$ with the property that the restriction to $X$ of each function in $\operatorname{VMO}(\mathbf{C})$, which is holomorphic on $X$, is the limit of a sequence of rational functions with poles off $X$. Our conditions involve the notion of onedimensional Hausdorff content. As an application, a result related to the inner boundary conjecture is proven.
\end{abstract}

0. Introduction and statement of results. Let $X$ be a compact subset of the complex plane $\mathbf{C}$, and let $\partial_{i} X$ denote its inner boundary, that is, the set of points in $\partial X$ which are not in the closure of a single connected component of $\mathbf{C} \backslash X$. The starting point of this paper is the following theorem.

THEOREM 0. Let $X \subset \mathbf{C}$ be compact and assume that $\partial_{i} X$ has finite onedimensional Hausdorff measure. Let $f$ be a continuous function on the Riemann sphere which is holomorphic on $X$. Then we can find a sequence of functions $f_{n}$ in $\mathrm{BMO}(\mathbf{C})$, each holomorphic in a neighborhood of $X$, such that $f_{n} \rightarrow f$ in $\mathrm{BMO}(\mathbf{C})$.

To explain the motivation for the above result let us begin by recalling that the problem of (qualitative) uniform rational approximation is controlled by a set function $\alpha$ called continuous analytic capacity (see [3] for its definition and basic properties). It turns out that no metric description of $\alpha$ is known, so that geometric sufficient conditions for rational approximation have been lacking and a number of natural conjectures are still open. To get precise statements for two of them let us recall the definition of the two classical algebras $R(X)$ and $A(X)$ associated to the compact $X$. The latter is the set of continuous functions on $X$ which are holomorphic on $\stackrel{X}{X}$, and the former is the uniform closure in $C(X)$ of the rational functions with poles off $X$. Vitushkin discovered that, in the problem of deciding whether $R(X)=A(X)$, a decisive role is played by the structure of $\partial_{i} X$. Accordingly he formulated $[\mathbf{1 9}]$ the so-called

INNER BOUNDARY CONJECTURE. If $\alpha\left(\partial_{i} X\right)=0$, then $R(X)=A(X)$.

It is by now clear that the inner boundary conjecture is closely linked to the difficult problem of the semiadditivity of $\alpha$ (see [2]). To get rid of the complications

Received by the editors May 16, 1984.

1980 Mathematics Subject Classification (1985 Revision). Primary 30E10; Secondary 42B99.

Key words and phrases. Rational function, approximation, BMO, Hausdorff content.

Partially supported by a Fulbright/MEC Grant. 
inherent to the semiadditivity problem, Melnikov and Vitushkin have considered the following weaker form of the inner boundary conjecture [12].

THE FINITE LENGTH CONJECTURE. If $\partial_{i} X$ has finite one-dimensional Hausdorff measure, then $R(X)=A(X)$.

Thus, clearly, Theorem 0 is a weak form of the finite length conjecture. The reader will realize later that the space $\mathrm{BMO}$ comes naturally into the scene through its relation with one-dimensional Hausdorff content (and, consequently, with onedimensional Hausdorff measure).

It might be interesting to remark at this point that the finite length conjecture is known to be true when $\partial_{i} X$ is a $C^{1+\varepsilon}$ Jordan arc [19] but, to the best of our knowledge, it is still unsolved even when $\partial_{i} X$ is a $C^{1}$ Jordan arc, or when $\partial_{i} X$ is the $1 / 4$ planar Cantor set $[6,7]$, the simplest example of (Besicovitch) irregular set.

Motivated by Theorem 0 and by a recent result of Wolff giving a natural definition of the space BMO on a compact subset of $\mathbf{C}$, we proceed to study the general problem of BMO rational approximation. Before stating our main results it is convenient to introduce some notation and terminology which will be used later.

Let $m$ be Lebesgue measure on $\mathbf{C}$. Let us fix $f \in L_{\text {loc }}^{1}(\mathbf{C})$. The mean oscillation of $f$ on a disc $\Delta$ is

$$
\Omega(f, \Delta)=\frac{1}{|\Delta|} \int_{\Delta}\left|f(z)-f_{\Delta}\right| d m(z),
$$

where $|\Delta|=m(\Delta)$ is the area of $\Delta$ and $f_{\Delta}=(1 /|\Delta|) \int_{\Delta} f(z) d m(z)$ is the mean value of $f$ on $\Delta$. We write $f \in \operatorname{BMO}(\mathbf{C})$ and we say that $f$ is of bounded mean oscillation if

$$
\|f\|_{*}=\sup _{\Delta} \Omega(f, \Delta)<\infty
$$

the supremum being taken over all discs $\Delta$. The functional \|\|$_{*}$ is a (complete) seminorm on $\operatorname{BMO}(\mathbf{C})$ vanishing only on constant functions. For $f$ in $\operatorname{BMO}(\mathbf{C})$ and $\delta>0$, set

$$
\Omega_{f}(\delta)=\sup \{\Omega(f, \Delta): \text { radius } \Delta \leq \delta\} .
$$

The space $\operatorname{VMO}(\mathbf{C})$ is the set of those functions $f \in \mathrm{BMO}(\mathbf{C})$ which have vanishing mean oscillation, that is, which satisfy $\Omega_{f}(\delta) \rightarrow 0$ as $\delta \rightarrow 0^{+}$.

Let us fix now a compact $X \subset \mathrm{C}$. Define

$$
\operatorname{BMO}(X)=\left\{f_{\mid X}: f \in \operatorname{BMO}(\mathbf{C})\right\}
$$

and, for $f \in \operatorname{BMO}(X)$, write

$$
\|f\|_{X}=\inf \left\{\|F\|_{*}: F=f \text { almost everywhere on } X\right\}
$$

and

$$
\|f\|_{\mathrm{BMO}(X)}=\left|\int_{X} f(z) d m(z)\right|+\|f\|_{X},
$$

so that \|\|$_{\mathrm{BMO}(X)}$ is a Banach space norm on $\operatorname{BMO}(X)$. See $\S 1$ below for a discussion of Wolff's intrinsic description of $\mathrm{BMO}(X)$ and of \|\|$_{X}$.

Let $R_{*}(X)$ denote the closure in $\mathrm{BMO}(X)$ of the algebra of rational functions with poles off $X$, and let $\mathrm{VMO}_{a}(X)$ denote the space of restrictions to $X$ of those functions in $\operatorname{VMO}(\mathbf{C})$ which are holomorphic on $\stackrel{\circ}{X}$. 
Write $M^{1}(E)$ (respectively $M_{*}^{1}(E)$ ) for the one-dimensional (respectively lower one-dimensional) Hausdorff content of the set $E \subset \mathbf{C}$ (see $\S 1$ for definitions). Finally, $\Delta(z, \delta)$ is the open disc with center $z$ and radius $\delta$, and $\nabla$ means gradient.

We can now state our main results.

THEOREM 1. Let $X \subset \mathbf{C}$ be compact and let $f \in \operatorname{VMO}(\mathbf{C})$. Then the following are equivalent.

(i) $f_{\mid X} \in R_{*}(X)$.

(ii) There exists a constant $C>0$ such that

$$
\left|\int \frac{\partial \phi}{\partial \bar{\xi}}(\xi) f(\xi) d m(\xi)\right| \leq C \delta\|\nabla \phi\|_{\infty} \Omega_{f}(\delta) M^{1}(\Delta(z, \delta) \backslash X)
$$

for all $z$, all $\delta>0$ and all continuously differentiable functions $\phi$ supported on $\Delta(z, \delta)$.

(iii) There exist $r \geq 1, \delta_{0}>0$ and a function $a(\delta)$, with $a(\delta) \rightarrow 0$ as $\delta \rightarrow 0^{+}$, such that

$$
\left|\int \frac{\partial \phi}{\partial \bar{\xi}}(\xi) f(\xi) d m(\xi)\right| \leq \delta\|\nabla \phi\|_{\infty} a(\delta) M^{1}(\Delta(z, r \delta) \backslash X)
$$

for all $z$, all $\delta \leq \delta_{0}$ and all continuously differentiable functions $\phi$ supported on $\Delta(z, \delta)$.

THEOREM 2. Let $X \subset \mathbf{C}$ be compact. Then the following are equivalent.

(i) $\mathrm{VMO}_{a}(X) \subset R_{*}(X)$.

(ii) There exists an absolute constant $C>0$ such that

$$
M_{*}^{1}(\Delta(z, \delta) \backslash \stackrel{\circ}{X}) \leq C M^{1}(\Delta(z, \delta) \backslash X)
$$

for all $z$ and all $\delta>0$.

(iii) There exist $r \geq 1, \delta_{0}>0$ and $C>0$ such that

$$
M_{*}^{1}(\Delta(z, \delta) \backslash \stackrel{\circ}{X}) \leq C M^{1}(\Delta(z, r \delta) \backslash X)
$$

for all $z$ and all $\delta \leq \delta_{0}$.

COROLlaRY. Let $X$ be a compact nowhere dense subset of $\mathbf{C}$. Then $C(X) \subset$ $R_{*}(X)$ if and only if there exist $r \geq 1, \delta_{0}>0$ and $C>0$ such that

$$
M^{1}(\Delta(z, r \delta) \backslash X) \geq C \delta
$$

for all $z$ and all $\delta \leq \delta_{0}$.

To prove the corollary from Theorem 2 simply recall that $2 \delta \geq M_{*}^{1}(\Delta(z, \delta)) \geq \delta$ for all $z$, and that $C(X)$ is dense in $\mathrm{VMO}_{a}(X)$ (in the norm of $\mathrm{BMO}(X)$ ) whenever $X$ has no interior.

Notice that our results are formally similar to Vitushkin's theorems on uniform rational approximation [3, Chapter VIII] and to O'Farrell's theorems on Lip $\alpha$ rational approximation $[\mathbf{1 3}, \mathbf{1 4}]$. In fact, since $B M O$ can be viewed as the natural limit of the $\operatorname{Lip} \alpha$ spaces as $\alpha \rightarrow 0^{+}$, Theorems 1 and 2 can also be interpreted as limiting statements of O'Farrell's results.

In the proofs, we shall depend heavily on Vitushkin's constructive scheme for approximation and on two notions of capacity, related to BMO and VMO respectively, which play a role analogous to that of analytic capacity $\gamma$ and of continuous 
analytic capacity $\alpha$ in uniform rational approximation theory. The main difference, as in the $\operatorname{Lip} \alpha$ case, is that our capacities have a metrical nature and, consequently, are semiadditive. However, we use the metrical description of capacities only at the end of the proof, to restate our results in terms of Hausdorff contents.

One of the technical problems we must face comes from the fact that we cannot use the classical method to match the second coefficient (in Taylor's expansion at $\infty$ ) of certain localized functions, because BMO is not an algebra. Following a recent idea of Lindberg [10] we construct functions with prescribed second coefficients at $\infty$ using Beurling transforms, and then we control their BMO norms appealing to the general fact that Caldéron-Zygmund operators of homogeneous type map $L^{\infty}$ into BMO continuously. Finally, it should be mentioned that we do not know if the inclusion $R_{*}(X) \subset \mathrm{VMO}_{a}(X)$ is always true. This would follow from an appropriate VMO version of Wolff's extension theorem.

The paper is organized as follows. In $\S 1$, we state and discuss the basic definitions and results we will need in the sequel. In $\S 2$, we introduce the capacities relevant to our problem, and we show that they are related to one-dimensional Hausdorff contents. In $\S 3$ we prove the necessary conditions for approximation in Theorems 1 and 2 , and in $\S 4$ the sufficient ones. A notion of BMO analytically negligible set is introduced in $\S 5$ and, as an application, an improved version of Theorem 0 is proven. In $\S 6$ we approximate $\operatorname{Lip} \alpha$ functions by rational functions in BMO norms and, finally, in $\S 7$ we present some examples.

\section{Basic definitions and results.}

A. If $\Delta$ is a disc and $f \in \operatorname{BMO}(\mathbf{C})$, then we set

$$
\|f\|_{*, \Delta}=\sup \{\Omega(f, B): B \operatorname{disc} \subset \Delta\} .
$$

We frequently use the following elementary lemma.

1.1. LemMA. Let $\Delta$ and $\Delta^{*}$ be discs of radii $\delta$ and $\delta^{*}$ respectively. If $\Delta^{*} \subset \Delta$, then

(i) $\Omega\left(f, \Delta^{*}\right) \leq 2\left(\delta / \delta^{*}\right)^{2} \Omega(f, \Delta)$,

(ii) $\left|f_{\Delta^{*}}-f_{\Delta}\right| \leq C \log \left(\delta / \delta^{*}\right)\|f\|_{*, \Delta}$ for some absolute constant $C$.

For (i) see [18, Lemma 2, p. 596] and for (ii) see [5, 1.1, p. 223].

B. The space $\mathrm{BMO}(X)$. For a (Lebesgue) measurable subset $X$ of $\mathbf{C}$ define $\operatorname{BMO}(X)$ and $\|f\|_{X}$ as in (1) and (2). We have the following intrinsic description of $\mathrm{BMO}(X)$.

THEOREM (WOLFF [20]). Let $f$ be a complex measurable function on $X$. Then the following are equivalent.

(i) $f \in \operatorname{BMO}(X)$.

(ii) There exist $\lambda>0$ and $C>0$ such that for each disc $\Delta$

$$
\frac{1}{|\Delta|} \int_{\Delta \cap X} \exp \left(\lambda\left|f(z)-f_{\Delta \cap X}\right|\right) d m(z) \leq C,
$$

where

$$
f_{\Delta \cap X}=\frac{1}{|\Delta||X|} \int_{\Delta \cap X} f(z) d m(z)
$$


(iii) There exist $\lambda>0$ and $C>0$ such that for each disc $\Delta$ one can find a constant $c_{\Delta}$ so that

$$
\frac{1}{|\Delta|} \int_{\Delta \cap X} \exp \left(\lambda\left|f(z)-c_{\Delta}\right|\right) d m(z) \leq C .
$$

Given $f$ in $\operatorname{BMO}(X)$, define $\Lambda_{1}(f, X)$ as the set of those positive $\lambda$ satisfying

$$
\frac{1}{|\Delta|} \int_{\Delta \cap X} \exp \left(\lambda\left|f(z)-f_{\Delta \cap X}\right|\right) d m(z) \leq 2
$$

and define $\Lambda_{2}(f, X)$ as the set of those positive $\lambda$ with the property that for each disc $\Delta$ there is a constant $c_{\Delta}$ with

$$
\frac{1}{|\Delta|} \int_{\Delta \cap X} \exp \left(\lambda\left|f(z)-c_{\Delta}\right|\right) d m(z) \leq 2
$$

Set

$$
\lambda_{j}(f, X)=\inf \left\{\lambda^{-1}: \lambda \in \Lambda_{j}(f, X)\right\}, \quad j=1,2 .
$$

The proof of the above theorem shows that

$$
C^{-1} \lambda_{j}(f, X) \leq\|f\|_{X} \leq C \lambda_{j}(f, X), \quad j=1,2,
$$

for some absolute constant $C>1$.

C. Hausdorff content. A measure function is an increasing function $h(t), t \geq 0$, such that $\lim _{t \rightarrow 0} h(t)=0$. If $h$ is a measure function and $E \subset \mathbf{C}$ we write

$$
M^{h}(E)=\inf \sum_{j} h\left(\delta_{j}\right)
$$

where the infimum is taken over all countable coverings of $E$ by squares $Q_{j}$ with sides of length $\delta_{j}$, parallel to the coordinate axis. When $h(t)=t^{\beta}, 0<\beta$, $M^{h}(E)=M^{\beta}(E)$ is called the $\beta$-dimensional Hausdorff content of $E$. The lower one-dimensional Hausdorff content of $E$ is defined by

$$
M_{*}^{1}(E)=\sup M^{h}(E),
$$

the supremum being taken over all measure functions $h$ which satisfy $h(t) \leq t$ and $h(t) t^{-1} \rightarrow 0$ as $t \rightarrow 0^{+}$.

D. Let $f$ be a compactly supported function in $L^{\infty}(\mathbf{C})$. Then the Beurling transform of $f$ is the function

$$
(B f)(z)=\lim _{\varepsilon \rightarrow 0} \int_{|\xi|>\varepsilon} \frac{f(z-\xi)}{\xi^{2}} d m(\xi) .
$$

It is well known that the limit in the right-hand side exists for $(m)$ almost all $z$ $\left[16,5.4\right.$, p. 317], and that $\|B f\|_{*} \leq C\|f\|_{\infty}$ for some absolute constant $C[16,4.4$, p. 308]. We observe also that for $|z|$ large we have the expansion

$$
(B f)(z)=\left(\int f(\xi) d m(\xi)\right) z^{-2}+\cdots .
$$

E. We denote by $C_{0}^{1}(D)$ the set of all continuously differentiable functions on $\mathbf{C}$ which have compact support contained in $D$. For $\phi \in C_{0}^{1}(\mathbf{C})$ we set

$$
\bar{\partial} \phi=\frac{\partial \phi}{\partial \bar{z}}=\frac{1}{2}\left(\frac{\partial \phi}{\partial x}+i \frac{\partial \phi}{\partial y}\right) \text {. }
$$

If $\Delta=\Delta(z, \delta)$ and $r>0$ we let $r \Delta$ stand for $\Delta(z, r \delta)$.

The letter $C$ will denote a positive constant, independent of the relevant variables under consideration, which may be different at each occurrence. 
2. Capacities. Let $K \subset \mathbf{C}$ be compact. Let $B(K)$ (respectively $V(K)$ ) be the set of functions $f$ in $\operatorname{BMO}(\mathbf{C})$ (respectively in $\operatorname{VMO}(\mathbf{C})$ ) such that $\|f\|_{*} \leq 1$ and $f$ is holomorphic in $\mathbf{C} \backslash K$. Since for $f \in \operatorname{BMO}(\mathbf{C})$ one has $[\mathbf{1 6}, 3.9$, p. 222]

$$
\int_{\mathbf{C}} \frac{|f(z)|}{1+|z|^{3}} d m(z)<\infty,
$$

each $f$ in $B(K)$ is also holomorphic at $\infty$. Thus we can define

$$
\gamma_{*}(K)=\sup \left\{\left|f^{\prime}(\infty)\right|: f \in B(K)\right\}
$$

and

$$
\alpha_{*}(K)=\sup \left\{\left|f^{\prime}(\infty)\right|: f \in V(K)\right\}
$$

If $E$ is an arbitrary subset of $\mathbf{C}$, we set

$$
\begin{aligned}
& \gamma_{*}(E)=\sup \left\{\gamma_{*}(K): K \text { compact } \subset E\right\} \\
& \alpha_{*}(E)=\sup \left\{\alpha_{*}(K): K \text { compact } \subset E\right\} .
\end{aligned}
$$

Proposition. There exists an absolute constant $C>0$ such that $C^{-1} M^{1}(E) \leq$ $\gamma_{*}(E) \leq C M^{1}(E)$ and

$$
C^{-1} M_{*}^{1}(E) \leq \alpha_{*}(E) \leq C M_{*}^{1}(E)
$$

whenever $E$ is $\sigma$-compact.

Proof. We prove only (3). Suppose first that $E$ is compact. Consider a measure function $h(t)$, with $h(t) \leq t$ and $h(t) t^{-1} \rightarrow 0$ as $t \rightarrow 0^{+}$. By the Frostman Lemma $\left[4,1.5\right.$, p. 62] there exists a positive measure $\mu$ on $E$ such that $25 \mu(E) \geq M^{h}(E)$ and $\mu(\Delta(z, r)) \leq h(r), r>0, z \in \mathbf{C}$. Let $\hat{\mu}$ be the Cauchy transform of $\mu$; that is,

$$
\hat{\mu}(z)=\frac{1}{\pi} \int \frac{d \mu(\xi)}{z-\xi}, \quad z \in \mathbf{C} .
$$

It is proved in [9] that

$$
\|\hat{\nu}\|_{*} \leq C \sup _{z, r} \nu(\Delta(z, r)) r^{-1}
$$

for some absolute constant $C$ and for all compactly supported measures $\nu$. Hence $\|\hat{\mu}\|_{*} \leq C$. Moreover $\hat{\mu}$ is a $\operatorname{VMO}(\mathbf{C})$ function [9, Remark (iii), p. 370]. Since $\hat{\mu}$ is holomorphic outside $E$,

$$
M^{h}(E) \leq 25 \mu(E)=25(\hat{\mu})^{\prime}(\infty) \leq C \alpha_{*}(E),
$$

and so, taking supremum over $h$,

$$
M_{*}^{1}(E) \leq C \alpha_{*}(E) .
$$

To prove the reverse inequality, fix $f \in V(K)$. Consider a finite family $\left(Q_{j}\right)$ of (closed) dyadic squares which cover $E$. Let $r_{j}$ be the side length of $Q_{j}$ and $Q_{j}^{*}=\frac{3}{2} Q_{j}$ (the square of side $\frac{3}{2} r_{j}$ with the same center as $Q_{j}$ ). Then the topological boundary $\partial D$ of $D=\bigcup_{j} Q_{j}^{*}$ is a polygonal curve surrounding $K$. By a lemma of Harvey and Polking $\left[8,3.1\right.$, p. 43] there are functions $\phi_{j} \in C_{0}^{\infty}\left(Q_{j}^{*}\right)$ with $\sum \phi_{j}=1$ 
on a neighborhood of $\bigcup_{j} Q_{j}$ and $\left\|\nabla \phi_{j}\right\|_{\infty} \leq C r_{j}^{-1}$. If $\phi=1-\sum_{j} \phi_{j}$, Stokes' Theorem leads to

$$
\begin{aligned}
\left|f^{\prime}(\infty)\right| & =\left|\int_{\partial D} f(z) d z\right|=\left|\int_{\partial D} f(z) \phi(z) d z\right| \\
& =\left|\int_{D} f(z) \bar{\partial} \phi(z) d \bar{z} \wedge d z\right| \\
& \leq 2 \sum_{j}\left|\int_{Q_{j}^{*}} f(z) \bar{\partial} \phi(z) d m(z)\right| \\
& =2 \sum_{j}\left|\int_{Q_{j}^{*}}\left(f(z)-f_{Q_{j}^{*}}\right) \bar{\partial} \phi(z) d m(z)\right| \\
& \leq C \sum_{j} r_{j} M_{f}\left(\frac{3}{2} r_{j}\right) .
\end{aligned}
$$

Taking the infimum over all coverings by dyadic squares we obtain $[4,1.4$, p. 61] $\left|f^{\prime}(\infty)\right| \leq C M^{h}(E)$ where $h(t)=t M_{f}\left(\frac{3}{2} t\right)$. Thus $\alpha_{*}(E) \leq C M_{*}^{1}(E)$. Therefore (3) holds for $E$ compact. In [1] it is proved that if a set $E$ is the union of a countable family of subsets $\left(E_{n}\right)$ and $E_{n} \subset E_{n+1}$ for each $n$, then

$$
M^{h}(E) \leq C \lim _{n} M^{h}\left(E_{n}\right)
$$

for each measure function $h$ and some absolute constant $C$. Then (3) follows readily for $\sigma$-compact $E$ from the compact case.

3. Necessary conditions. Our first task is to obtain a BMO estimate for the Vitushkin localization operator. We need the following lemma.

3.1. LEMMA. Let $f$ be a compactly supported function in $\mathrm{BMO}(\mathbf{C})$, and assume spt $f \subset \Delta$ for some open disc $\Delta$. Then $\|f\|_{*} \leq C\|f\|_{*, 3 \Delta}$ for some absolute constant $C$.

Proof. Let $B$ be a disc. If $B \cap \Delta=\varnothing$ then $\Omega(f, B)=0$. So suppose $B \cap \Delta \neq \varnothing$ and consider two cases.

Case 1. $|B| \leq|\Delta|$. Then $B \subset 3 \Delta$, and thus $\Omega(f, B) \leq\|f\|_{*, 3 \Delta}$.

Case 2. $|B|>|\Delta|$. Let $\Delta_{1}$ be the disc with the same center as $\Delta$ such that $\left|\Delta_{1}\right|=$ $|B|$. Write $\Delta^{*}=3 \Delta_{1}$. Thus $B \subset \Delta^{*}$. Since $B$ and $\Delta^{*}$ have comparable radii, it follows from Lemma $1.1(\mathrm{i})$ that $\Omega(f, B) \leq C \Omega\left(f, \Delta^{*}\right)$. We estimate $\Omega\left(f, \Delta^{*}\right)$ by

$$
\begin{aligned}
\frac{2}{\left|\Delta^{*}\right|} \int_{\Delta^{*}}\left|f-f_{2 \Delta}\right| & =\frac{2}{\left|\Delta^{*}\right|} \int_{2 \Delta}\left|f-f_{2 \Delta}\right|+\frac{2}{\left|\Delta^{*}\right|} \int_{\Delta^{*} \backslash 2 \Delta}\left|f-f_{2 \Delta}\right| \\
& =\mathrm{I}+\mathrm{II} .
\end{aligned}
$$

The term $I$ is at most

$$
2 \Omega(f, 2 \Delta) \leq 2\|f\|_{*, 3 \Delta}
$$

and II is estimated as follows:

$$
\begin{aligned}
\mathrm{II} & =\frac{\left|f_{2 \Delta}\right|}{\left|\Delta^{*}\right|}\left|\Delta^{*} \backslash 2 \Delta\right| \leq\left|f_{2 \Delta}\right| \\
& =\frac{4}{3} \frac{1}{|2 \Delta|} \int_{2 \Delta \backslash \Delta}\left|f-f_{2 \Delta}\right| \leq \frac{4}{3} \Omega(f, 2 \Delta) \leq 2\|f\|_{*, 3 \Delta} .
\end{aligned}
$$


Recall that to each $\phi \in C_{0}^{1}(\mathbf{C})$ one associates the Vitushkin operator $T_{\phi}: L_{\text {loc }}^{1}(\mathbf{C})$ $\rightarrow L_{\text {loc }}^{1}(\mathbf{C})$, defined by

$$
\begin{aligned}
\left(T_{\phi} f\right)(z) & =\frac{1}{\pi} \int \frac{f(\xi)-f(z)}{\xi-z} \bar{\partial} \phi(\xi) d m(\xi) \\
& =\phi(z) f(z)+\frac{1}{\pi} \int \frac{f(\xi)}{\xi-z} \bar{\partial} \phi(\xi) d m(\xi) .
\end{aligned}
$$

For the basic properties of $T_{\phi}$ see $[3$, p. 210 and 2, p. 411].

3.2. Proposition. Let $\Delta$ be a disc of radius $\delta, \phi \in C_{0}^{1}(\Delta)$ and $f \in \operatorname{BMO}(\mathbf{C})$. Then

$$
\left\|T_{\phi} f\right\|_{*} \leq C \delta\|\nabla \phi\|_{\infty}\|f\|_{*, 3 \Delta}
$$

for some absolute constant $C$.

ProOF. Without loss of generality let us suppose that $\int_{3 \Delta} f=0$. Notice that

$$
T_{\phi} f=\phi f-\frac{1}{\pi}(f \bar{\partial} \phi) .
$$

By Kaufman's estimate (4)

$$
\left\|(f \bar{\partial} \phi)^{-}\right\|_{*} \leq C \sup _{z, r} r^{-1} \int_{\Delta(z, r)}|f \bar{\partial} \phi| .
$$

We now estimate $\int_{\Delta(z, r)}|f \bar{\partial} \phi|$ by

$$
\begin{aligned}
\|\nabla \phi\|_{\infty} \int_{\Delta(z, r) \cap \Delta}|f| & \leq\|\nabla \phi\|_{\infty}|\Delta(z, r) \cap \Delta|^{1 / 2}\left(\int_{\Delta(z, r) \cap \Delta}|f|^{2}\right)^{1 / 2} \\
& \leq C r\|\nabla \phi\|_{\infty}\left(\int_{3 \Delta}|f|^{2}\right)^{1 / 2} \\
& =C r\|\nabla \phi\|_{\infty}\left(\int_{3 \Delta}\left|f-f_{3 \Delta}\right|^{2}\right)^{1 / 2} \\
& \leq C r \delta\|\nabla \phi\|_{\infty}\|f\|_{*, 3 \Delta}
\end{aligned}
$$

where the last inequality follows from the John-Nirenberg Theorem $[16,3.12$, p. 227 .

Now let us estimate the BMO-norm of $\phi f$. By $3.1,\|\phi f\|_{*} \leq C\|\phi f\|_{*, 3 \Delta}$. If $B$ is a disc of radius $r$ and $B \subset 3 \Delta$, then

$$
\begin{aligned}
& \frac{1}{|B|} \int_{B}\left|\phi f-\phi_{B} f_{B}\right| \\
& \quad \leq \frac{1}{|B|} \int_{B}|\phi|\left|f-f_{B}\right|+\frac{1}{|B|} \int\left|f_{B}\right|\left|\phi-\phi_{B}\right| \\
& \quad \leq \delta\|\nabla \phi\|_{\infty}\|f\|_{*, 3 \Delta}+r\left|f_{B}\right|\|\nabla \phi\|_{\infty} .
\end{aligned}
$$

From 1.1(ii) we obtain

$$
r\left|f_{B}\right|=r\left|f_{B}-f_{3 \Delta}\right| \leq C r \log (\delta / r)\|f\|_{*, 3 \Delta} \leq C \delta\|f\|_{*, 3 \Delta},
$$

and thus

$$
\|\phi f\|_{*} \leq C \delta\|\nabla \phi\|_{\infty}\|f\|_{*, 3 \Delta} .
$$

We still need another lemma. 
3.3. LemMA. Let $f \in \operatorname{VMO}(\mathbf{C})$, let $X \subset \mathbf{C}$ be compact and $\varepsilon>0$. Then there is an open set $U$ (depending on $f, X$ and $\varepsilon$ ) containing $X$ such that $\|f\|_{U} \leq$ $C\|f\|_{X}+\varepsilon, C$ being an absolute positive constant.

PROOF. Write

$$
U_{d}=\{z: d(z, X)<d\}, \quad d>0 .
$$

Since a function in $\operatorname{VMO}(\mathbf{C})$ is the limit, in $\mathrm{BMO}(\mathbf{C})$, of a sequence of (uniformly) continuous functions in $\operatorname{BMO}(\mathbf{C})[\mathbf{1 7}$, Theorem 1 , p. 392], we can suppose that $f$ is bounded on $U_{1}$.

Consider a positive $\lambda$ in $\Lambda_{1}(f, X)$ with $\lambda^{-1}<\lambda_{1}(f, X)+\varepsilon$. Since $f \in \operatorname{VMO}(\mathbf{C})$, there exists $\eta>0$ such that

$$
\frac{1}{|\Delta|} \int_{\Delta} \exp \left(\lambda\left|f-f_{\Delta}\right|\right) \leq 2
$$

whenever $\Delta$ is a disc of radius at most $\eta$. Fix now a disc $\Delta$ of radius $\delta$, and distinguish two cases.

Case 1. $\delta<\eta$. Set $c_{\Delta}=f_{\Delta}$. For $d>0$ we then have

$$
\frac{1}{|\Delta|} \int_{\Delta \cap U_{d}} \exp \left(\lambda\left|f-c_{\Delta}\right|\right) \leq \frac{1}{|\Delta|} \int_{\Delta} \exp \left(\lambda\left|f-f_{\Delta}\right|\right) \leq 2 \text {. }
$$

Case 2. $\delta \geq \eta$. Set $c_{\Delta}=f_{\Delta \cap X}$. For $0<d \leq 1$ we obtain

$$
\begin{aligned}
& \frac{1}{|\Delta|} \int_{\Delta \cap U_{d}} \exp \left(\frac{\lambda}{2}\left|f-c_{\Delta}\right|\right) \\
& \quad \leq \frac{1}{|\Delta|} \int_{\Delta \cap X} \exp \left(\frac{\lambda}{2}\left|f-c_{\Delta}\right|\right)+\frac{1}{|\Delta|} \int_{U_{d} \backslash X} \exp \left(\frac{\lambda}{2}\left|f-c_{\Delta}\right|\right) \\
& \quad=\mathrm{I}+\mathrm{II} .
\end{aligned}
$$

We easily get

$$
\mathrm{II} \leq \frac{\left|U_{d} \backslash X\right|}{\pi \eta^{2}} \exp \left(\lambda\|f\|_{L^{\infty}\left(U_{1}\right)}\right)
$$

which is at most $2-\sqrt{ } 2$ if $d$ is small enough. We estimate I by

$$
\left(\frac{|\Delta \cap X|}{|\Delta| \cdot} \frac{1}{|\Delta|} \int_{\Delta \cap X} \exp \left(\lambda\left|f-c_{\Delta}\right|\right)\right)^{1 / 2} \leq \sqrt{ } 2
$$

Therefore, for each disc $\Delta$ there exists a constant $c_{\Delta}$ such that

$$
\frac{1}{|\Delta|} \int_{\Delta \cap U_{d}} \exp \left(\frac{\lambda}{2}\left|f-c_{\Delta}\right|\right) \leq 2
$$

if $d$ is sufficiently small. Hence

$$
\lambda_{2}\left(f, U_{d}\right) \leq 2 / \lambda \leq 2\left(\lambda_{1}(f, X)+\varepsilon\right)
$$

and so

$$
\|f\|_{U_{d}} \leq C \lambda_{2}\left(f, U_{d}\right) \leq C \lambda_{1}(f, X)+C \varepsilon \leq C\|f\|_{X}+C \varepsilon .
$$

This completes the proof.

Proof OF (i) $\Rightarrow$ (ii) IN THEOREM 1 . We are going to prove that, for some absolute constant $C$,

$$
\left|\int \bar{\partial} \phi(\xi) f(\xi) d m(\xi)\right| \leq C \delta\|\nabla \phi\|_{\infty} \Omega_{f}(\delta) \gamma_{*}(\Delta \backslash X)
$$


for all discs $\Delta$ of radius $\delta$ and for all $\phi \in C_{0}^{1}(\Delta)$. This implies (ii) in view of the proposition in $\S 2$.

To show (5), take a sequence $\left(f_{n}\right)$ of functions in $C_{0}^{\infty}(\mathbf{C})$ such that each $f_{n}$ is holomorphic in a neighborhood of $X$ and $f_{n \mid X} \rightarrow f_{\mid X}$ in $\operatorname{BMO}(X)$. By Lemma 3.3 there exists an open set $U_{n} \supset X$ with

$$
\left\|f_{n}-f\right\|_{U_{n}} \leq C\left\|f_{n}-f\right\|_{X}+1 / n .
$$

The definition of the norm in $\operatorname{BMO}\left(U_{n}\right)$ guarantees that there exist functions $D_{n}$ in $\mathrm{BMO}(\mathbf{C})$ satisfying $D_{\left.n\right|_{U_{n}}}=f_{n}-f$ and

$$
\left\|D_{n}\right\|_{*} \leq C\left\|f_{n}-f\right\|_{X}+2 / n \text {. }
$$

If we set $h_{n}=D_{n}+f$, then $h_{n} \in \mathrm{BMO}(\mathbf{C}), h_{n_{\mid U_{n}}}=f_{n}$ and $h_{n} \rightarrow f$ in $\operatorname{BMO}(\mathbf{C})$. Since $h_{n}$ is holomorphic in a neighborhood of $X, T_{\phi} h_{n}$ is holomorphic outside a compact subset of $\Delta \backslash X$. Thus

$$
\begin{aligned}
\left|\int \bar{\partial} \phi(\xi) h_{n}(\xi) d m(\xi)\right| & =\left|\left(T_{\phi} h_{n}\right)^{\prime}(\infty)\right| \leq\left\|T_{\phi} h_{n}\right\|_{*} \gamma_{*}(\Delta \backslash X) \\
& \leq C \delta\|\nabla \phi\|_{\infty}\left\|h_{n}\right\|_{*, 3 \Delta} \gamma_{*}(\Delta \backslash X)
\end{aligned}
$$

by Lemma 3.2. Letting $n \rightarrow \infty$,

$$
\begin{aligned}
\left|\int \bar{\partial} \phi(\xi) f(\xi) d m(\xi)\right| & \leq C \delta\|\nabla \phi\|_{\infty}\|f\|_{*, 3 \Delta \gamma_{*}}(\Delta \backslash X) \\
& \leq C \delta\|\nabla \phi\|_{\infty} \Omega_{f}(3 \delta) \gamma_{*}(\Delta \backslash X) .
\end{aligned}
$$

Finally, observe that $\Omega_{f}(3 \delta) \leq C \Omega_{f}(\delta)$ by a result of Spanne $[\mathbf{1 8}$, Lemma 2, p. 596].

The implication (i) $\Rightarrow$ (ii) in Theorem 2 follows from a similar argument.

\section{Sufficient conditions.}

4.1. LEMMA (DECAY ESTIMATE). Let $\Delta=\Delta(a, \delta)$ be an open disc, and suppose that $f \in L_{\mathrm{loc}}^{1}(\mathbf{C})$ is holomorphic outside a compact subset of $\Delta$ and $f(\infty)=$ 0 . Then, for $|z-a| \geq 2 \delta$,

$$
|f(z)| \leq C \frac{\delta}{d(z, \Delta)} \Omega(f, 2 \Delta),
$$

$C$ being an absolute constant.

Proof. Let $\Delta^{*}=\frac{3}{2} \Delta$ and consider $\phi \in C_{0}^{1}\left(\Delta^{*}\right),\|\nabla \phi\|_{\infty} \leq C / \delta, \phi=1$ on $\Delta$. If $|z-a| \geq 2 \delta$, then, from Stokes' Theorem and the Cauchy-Pompeiu formula

$$
-\frac{1}{\pi} \int \frac{\bar{\partial} \phi(\xi)}{\xi-z} d m(\xi)=\phi(z)=0
$$

we get

$$
\begin{aligned}
f(z) & =\frac{1}{2 \pi i} \int_{\partial \Delta^{*}} \frac{f(\xi)}{\xi-z} d \xi=\frac{1}{2 \pi i} \int_{\partial \Delta^{*}}(1-\phi(\xi)) \frac{f(\xi)}{\xi-z} d \xi \\
& =-\frac{1}{2 \pi i} \int_{\Delta^{*}} \bar{\partial} \phi(\xi) \frac{f(\xi)}{\xi-z} d \bar{\xi} \wedge d \xi \\
& =-\frac{1}{\pi} \int_{\Delta^{*}} \frac{\bar{\partial} \phi(\xi)}{\xi-z}\left(f(\xi)-f_{\Delta^{*}}\right) d m(\xi) .
\end{aligned}
$$


Thus

$$
\begin{aligned}
|f(z)| & \leq \frac{C}{\delta d(z, \Delta)} \int_{\Delta^{*}}\left|f(\xi)-f_{\Delta^{*}}\right| d m(\xi) \\
& =C \frac{\delta}{d(z, \Delta)} \Omega\left(f, \Delta^{*}\right) \leq C \frac{\delta}{d(z, \Delta)} \Omega(f, 2 \Delta) .
\end{aligned}
$$

If $f$ is holomorphic on a neighborhood of $\infty, f(\infty)=0$, and $a \in \mathbf{C}$, then $f$ admits a Taylor expansion

$$
f(z)=\frac{a_{1}}{z-a}+\frac{a_{2}}{(z-a)^{2}}+\cdots
$$

for $|z|$ sufficiently large. Define $\beta(f, a)=a_{2}$.

4.2. Lemma (SECOND COEFFICIENT ESTIMATE). Let $f \in \operatorname{BMO}(\mathbf{C})$ be holomorphic outside a compact subset $K$ of an open disc $\Delta=\Delta(a, \delta)$. Then

$$
|\beta(f, a)| \leq C \delta\|f\|_{*} \gamma_{*}(K)
$$

for some absolute constant $C$.

Proof. Let $\phi \in C_{0}^{1}(2 \Delta),\|\nabla \phi\|_{\infty} \leq C / \delta, \phi=1$ on a neighborhood of $K$. Then $f=T_{\phi} f+f(\infty)$. If we set

$$
F(\xi)=(\xi-a) f(\xi), \quad \xi \in \mathbf{C},
$$

then

$$
\beta(f, a)=\frac{1}{\pi} \int \bar{\partial} \phi(\xi)(\xi-a) f(\xi) d m(\xi)=\left(T_{\phi} F\right)^{\prime}(\infty) .
$$

Hence, by 3.2 and the definition of $\gamma_{*}$,

$$
|\beta(f, a)| \leq C\|F\|_{*, 6 \Delta \gamma_{*}}(K) .
$$

To complete the proof simply observe that, by the argument used in the proof of 3.2 ,

$$
\|F\|_{*, 6 \Delta} \leq C \delta\|f\|_{*} .
$$

PROOF OF (iii) $\Rightarrow$ (i) IN THEOREM 1. By hypothesis and the proposition in $\S 2$, there exist $r \geq 1$ and a function $a(\delta)$, with $a(\delta) \rightarrow 0$ as $\delta \rightarrow 0^{+}$, such that

$$
\left|\int \bar{\partial} \phi(\xi) f(\xi) d m(\xi)\right| \leq \delta\|\nabla \phi\|_{\infty} a(\delta) \gamma_{*}(r \Delta \backslash X)
$$

for each disc $\Delta$ of radius $\delta$ and each $\phi \in C_{0}^{1}(\Delta)$. Fix $\delta>0$, and let $\left(\Delta_{j}\right),\left(\phi_{j}\right)$ be a scheme for the approximation $\left[3\right.$, p. 211] corresponding to $\delta$. That is, $\left(\Delta_{j}\right)$ is a countable family of open discs of radius $\delta$ which cover $\mathbf{C}$ and such that each $z \in \mathbf{C}$ belongs to at most 21 discs, and $\left(\phi_{j}\right)$ is a family of functions satisfying $\phi_{j} \in C_{0}^{1}\left(\Delta_{j}\right), 0 \leq \phi_{j} \leq 1,\left\|\nabla \phi_{j}\right\|_{\infty} \leq C \delta^{-1}$ and $\sum_{j} \phi_{j}=1$. Write $f_{j}=T_{\phi_{j}} f$. Let $J_{0}=\left\{k: \Delta_{k} \cap X \neq \varnothing\right\}$ and let $J$ be the set of those indexes $j$ such that there is $k \in J_{0}$ with $\Delta_{k} \cap \Delta_{j} \neq \varnothing$. Let $\sum^{\prime}$ indicate summation over the indexes $j$ belonging to $J$. Then $\sum^{\prime} \phi_{j}=1$ on $\bigcup_{k \in J_{0}} \Delta_{k} \supset X$. If $F=\sum^{\prime} f_{j}$ and $h=f-F$ then $f=h+F$ and $h$ is holomorphic on a neighborhood of $X$. By (6),

$$
\left|f_{j}^{\prime}(\infty)\right|=\left|\frac{1}{\pi} \int \bar{\partial} \phi_{j}(\xi) f(\xi) d m(\xi)\right| \leq C a(\delta) \gamma_{*}\left(r \Delta_{j} \backslash X\right) .
$$


Let $g_{j}$ be a function in $\mathrm{BMO}(\mathbf{C})$ such that $\left\|g_{j}\right\|_{*} \leq C a(\delta), g_{j}$ is holomorphic outside a compact subset of $r \Delta_{j} \backslash X, g_{j}(\infty)=0$ and $g_{j}^{\prime}(\infty)=f_{j}^{\prime}(\infty)$. We are going to see that

$$
\left\|F-\sum^{\prime} g_{j}\right\|_{*} \leq C b(\delta)
$$

where $b(\delta)=\Omega_{f}(3 \delta)+a(\delta)$. This will complete the proof, because $\sum^{\prime} g_{j}$ is holomorphic in a neighborhood of $X$ and $b(\delta) \rightarrow 0$ as $\delta \rightarrow 0^{+}$.

Since

$$
\left\|f_{j}-g_{j}\right\|_{*} \leq\left\|f_{j}\right\|_{*}+\left\|g_{j}\right\|_{*}, \quad\left\|g_{j}\right\|_{*} \leq C a(\delta)
$$

and

$$
\left\|f_{j}\right\|_{*}=\left\|T_{\phi_{j}} f\right\|_{*} \leq C \delta\left\|\nabla \phi_{j}\right\|_{\infty}\|f\|_{*, 3 \Delta_{j}} \leq C \Omega_{f}(3 \delta)
$$

inequality (7) follows from the next lemma.

4.3. LeMMA (DOUBLE ZERO LEMMA). Let $\left(\Delta_{j}\right)$ be a finite sequence of open discs of radius $\delta>0$, such that each $z \in \mathbf{C}$ belongs to at most $p$ discs $\Delta_{j}, p$ being a fixed positive integer. Suppose that $h_{j} \in \mathrm{BMO}(\mathbf{C})$ is holomorphic outside a compact subset of $\Delta_{j}$ and that $h_{j}$ has a double zero at $\infty$. Then, for some positive constant $C$ depending only on $p$,

$$
\left\|\sum_{j} h_{j}\right\|_{*} \leq C \max _{j}\left\|h_{j}\right\|_{*}
$$

PROOF. We claim that, without loss of generality, it is possible to assume that each $h_{j}$ has a triple zero at $\infty$. 4.2

To prove the claim set $\beta_{j}=\beta\left(h_{j}, z_{j}\right)$, where $z_{j}$ is the center of $\Delta_{j}$. By Lemma

$$
\left|\beta_{j}\right| \leq C \delta \gamma_{*}\left(\Delta_{j}\right)\left\|h_{j}\right\|_{*} \leq C \delta^{2}\left\|h_{j}\right\|_{*} .
$$

Let $\chi_{j}$ be the characteristic function of $2^{-1} \Delta_{j}$ and write

$$
\psi_{j}=\beta_{j} \chi_{j} /\left|2^{-1} \Delta_{j}\right| \quad \text { and } \quad b_{j}=B\left(\psi_{j}\right) .
$$

Thus

$$
\left\|b_{j}\right\|_{*} \leq C\left\|\psi_{j}\right\|_{\infty} \leq C\left\|h_{j}\right\|_{*}
$$

and

$$
\begin{aligned}
\left\|\sum_{j} b_{j}\right\|_{*} & =\left\|B\left(\sum_{j} \psi_{j}\right)\right\|_{*} \leq C\left\|\sum_{j} \psi_{j}\right\|_{\infty} \\
& \leq C \max _{j}\left\|\psi_{j}\right\|_{\infty} \leq C \max _{j}\left\|h_{j}\right\|_{*} .
\end{aligned}
$$

Since

$$
\left\|\sum_{j} h_{j}\right\|_{*} \leq\left\|\sum_{j}\left(h_{j}-b_{j}\right)\right\|_{*}+C \max _{j}\left\|h_{j}\right\|_{*}, \quad\left\|h_{j}-b_{j}\right\|_{*} \leq C\left\|h_{j}\right\|_{*},
$$

and $h_{j}-b_{j}$ has a triple zero at $\infty$, the claim is proven.

Suppose now that each $h_{j}$ has a triple zero at $\infty$. Fix a disc $B=\Delta(b, r)$. Write

$$
A_{n}=\{z: n \delta \leq|z-b|<(n+1) \delta\}, \quad n=0,1,2, \ldots
$$


Divide the indexes $j$ into classes $\left(z_{j}\right.$ is the center of $\left.\Delta_{j}\right) J_{n}=\left\{j: z_{j} \in A_{n}\right\}$. Then

$$
\# J_{n} \leq C n,
$$

$C$ being a constant which depends on $p$ only. Choose a positive integer $N$ such that $(N-1) \delta \leq r<N \delta$, and write

$$
\begin{aligned}
\Omega\left(\sum_{j} h_{j}, B\right) & \leq \sum_{j} \Omega\left(h_{j}, B\right) \\
& =\sum_{n=0}^{2 N} \sum_{j \in J_{n}} \Omega\left(h_{j}, B\right)+\sum_{n>2 N} \sum_{j \in J_{n}} \Omega\left(h_{j}, B\right) \\
& =\mathrm{I}_{N}+\mathrm{II}_{N} .
\end{aligned}
$$

Let us estimate $\mathrm{II}_{N}$. By (8)

$$
\mathrm{II}_{N} \leq C \sum_{n>2 N} n \max _{j \in J_{n}}\left\|h_{j}\right\|_{L^{\infty}(B)} .
$$

Fix $n>2 N$ and $j \in J_{n}$. Then $\left|z-z_{j}\right| \geq 2 \delta$ whenever $z \in B$. If $\left|z-z_{j}\right|=2 \delta$, then, by Lemma 4.1 ,

$$
\left|\left(\left(z-z_{j}\right) / \delta\right)^{3} h_{j}(z)\right| \leq C\left|h_{j}(z)\right| \leq C \Omega\left(h_{j}, 2 \Delta_{j}\right) .
$$

Since $h_{j}$ has a triple zero at $\infty$, it follows from the maximum modulus principle that

$$
\left|h_{j}(z)\right| \leq C\left(\delta /\left|z-z_{j}\right|\right)^{3} \Omega\left(h_{j}, 2 \Delta_{j}\right), \quad\left|z-z_{j}\right| \geq 2 \delta,
$$

and thus

$$
\left\|h_{j}\right\|_{L^{\infty}(B)} \leq C(n-N)^{-3}\left\|h_{j}\right\|_{*} .
$$

Therefore

$$
\mathrm{II}_{N} \leq C\left[\sum_{n>2 N} \frac{n}{(n-N)^{3}}\right] \max _{j}\left\|h_{j}\right\|_{*} \leq C \max _{j}\left\|h_{j}\right\|_{*} .
$$

Let us turn to $I_{N}$. For $N=1$ we obtain, according to (8),

$$
I_{1} \leq C \max _{j}\left\|h_{j}\right\|_{*} .
$$

Suppose $N \geq 2$ and fix $j \in J_{n}, 0 \leq n \leq 2 N$. Hence $3 N \Delta_{j} \supset B$. Since $3 N \Delta_{j}$ and $B$ have comparable radii,

$$
\Omega\left(h_{j}, B\right) \leq C \Omega\left(h_{j}, 3 N \Delta_{j}\right)
$$

by 1.1. It is now sufficient to show that

$$
\Omega\left(h_{j}, 3 N \Delta_{j}\right) \leq C N^{-2} \Omega\left(h_{j}, 2 \Delta_{j}\right)
$$

because then

$$
I_{N} \leq C N^{-2}\left(\sum_{n=0}^{2 N} n\right) \max _{j}\left\|h_{j}\right\|_{*} \leq C \max _{j}\left\|h_{j}\right\|_{*} .
$$


To simplify notation fix $j$ and set $h=h_{j}, \Delta=\Delta_{j}$ and $\Delta^{*}=3 N \Delta_{j}$. Then

$$
\begin{aligned}
\Omega\left(h, \Delta^{*}\right) & =\frac{1}{\left|\Delta^{*}\right|} \int_{\Delta^{*} \backslash 2 \Delta}\left|h-h_{\Delta \cdot}\right|+\frac{1}{\left|\Delta^{*}\right|} \int_{2 \Delta}\left|h-h_{\Delta^{*}}\right| \\
& =\mathrm{I}+\mathrm{II} .
\end{aligned}
$$

We estimate I by

$$
\frac{1}{\left|\Delta^{*}\right|} \int_{(2 \Delta)^{c}}|h|+\left|h_{\Delta^{*}}\right| .
$$

Since $h$ is holomorphic outside $\Delta$ and $h(\infty)=0$, the mean value of $h$ over the circle with the same center as $\Delta$ and radius $\rho>\delta$ is zero. From this we get, on the one hand, $\int_{\Delta^{*}} h=\int_{2 \Delta} h$, which gives

$$
h_{\Delta^{*}}=\left(4 / 9 N^{2}\right) h_{2 \Delta},
$$

and on the other hand

$$
3\left|h_{2 \Delta}\right|=\left|h_{\Delta}-h_{2 \Delta}\right| \leq 4 \Omega(h, 2 \Delta) .
$$

Therefore

$$
\left|h_{\Delta *}\right| \leq C N^{-2} \Omega(h, 2 \Delta) .
$$

We now estimate the first term in (10). Let $a$ be the center of $\Delta$. As before, using that $h$ has a triple zero at $\infty$, we obtain

$$
|h(z)| \leq C(\delta /|z-a|)^{3} \Omega(h, 2 \Delta), \quad|z-a| \geq 2 \delta .
$$

Thus

$$
\frac{1}{\left|\Delta^{*}\right|} \int_{(2 \Delta)^{c}}|h| \leq C N^{-2} \Omega(h, 2 \Delta) \delta \int_{2 \delta}^{\infty} \rho^{-2} d \rho=C N^{-2} \Omega(h, 2 \Delta) .
$$

Finally, we estimate II by $C N^{-2}\left(\Omega(h, 2 \Delta)+\left|h_{2 \Delta}-h_{\Delta} \cdot\right|\right)$. Therefore (9) is proven.

ProOF OF (iii) $\Rightarrow$ (i) IN THEOREM 2. Let $f \in \operatorname{VMO}(\mathbf{C})$ and suppose that $f$ is holomorphic on $\stackrel{\circ}{X}$. Let us check that condition (iii) in Theorem 1 is satisfied with $a(\delta)=\Omega_{f}(3 \delta)$. If $\Delta$ is a disc of radius $\delta$ and $\phi \in C_{0}^{1}(\Delta)$, then, since $T_{\phi} f$ is holomorphic outside $\Delta$ and on $\stackrel{\circ}{X}$,

$$
\begin{aligned}
\left|\int \bar{\partial} \phi(\xi) f(\xi) d m(\xi)\right| & =\pi\left|\left(T_{\phi} f\right)^{\prime}(\infty)\right| \\
& \leq \pi\left\|T_{\phi} f\right\|_{*} \alpha_{*}(\Delta \backslash \stackrel{\circ}{X}) \\
& \leq C \delta\|\nabla \phi\|_{\infty}\|f\|_{*, 3 \Delta} M_{*}^{1}(\Delta \backslash \stackrel{\circ}{X}) \\
& \leq C \delta\|\nabla \phi\|_{\infty} \Omega_{f}(3 \delta) M^{1}(r \Delta \backslash X) .
\end{aligned}
$$

\section{5. $\mathrm{BMO}$ analytically negligible sets.}

DEFINITION. We call a subset $E$ of $\mathbf{C}$ a BMO analytically negligible set if and only if each function $f \in \operatorname{VMO}(\mathbf{C})$ which is holomorphic on an open subset $U$ of $\mathbf{C}$ can be approximated in $\mathrm{BMO}(\mathbf{C})$ by functions which are holomorphic on $U$ and on a neighborhood of $E$. 
5.1. THEOREM. Let $E$ be a bounded $G_{\delta}$ subset of $\mathrm{C}$. Then $E$ is BMO analytically negligible if and only if $M_{*}^{1}(E)=0$.

Proof. Necessity. If $E$ is a BMO analytically negligible set then $\alpha_{*}(E)=0$. Suppose $M_{*}^{1}(E)>0$. Then, by $\left[1\right.$, Theorem 2, p. 11], $M_{*}^{1}(K)>0$ for some compact subset $K$ of $E$. But this is a contradiction because $M_{*}^{1}$ and $\alpha_{*}$ are comparable on compact sets. Thus $M_{*}^{1}(E)=0$.

Sufficiency. Let $U \subset \mathbf{C}$ be open and let $\Delta$ be an open disc. Then

$$
M_{*}^{1}(\Delta \backslash U) \leq M_{*}^{1}((\Delta \backslash U) \backslash E)+M_{*}^{1}(E)=M_{*}^{1}(\Delta \backslash(U \cup E)) .
$$

Since $\Delta \backslash(U \cup E)$ is $\sigma$-compact, because $E$ is $G_{\delta}$, we have by the proposition in $\S 2$

$$
\alpha_{*}(\Delta \backslash U) \leq C \alpha_{*}(\Delta \backslash(U \cup E)) \leq C \gamma_{*}(\Delta \backslash(U \cup E)) .
$$

Assume $f \in \operatorname{VMO}(\mathbf{C})$ to be holomorphic on $U$. If we apply to $f$ the approximation scheme of $\S 4$, we will get approximating functions with singularities in compact subsets of $(U \cup E)^{c}$, because of (12). This completes the proof.

We remark that, owing to well-known measure theoretic results, for Borel subsets $E$ of the plane $M_{*}^{1}(E)=0$ is equivalent to $E$ being of $\sigma$-finite length. Thus the following result contains Theorem 0 . In Example 3 in $\S 7$ it is shown that the $\sigma$-finite length hypothesis on the inner boundary is sharp.

5.2. CoROllary. Let $X \subset \mathrm{C}$ be compact and assume $M_{*}^{1}\left(\partial_{i} X\right)=0$. Then $\mathrm{VMO}_{a}(X) \subset R_{*}(X)$. In particular, $A(X) \subset R_{*}(X)$.

PROOF. Recall that for each $z \in \partial_{e} X=\partial X \backslash \partial_{i} X, \gamma(\Delta(z, \delta) \backslash X) \geq C \delta$ for $\delta$ small enough and for some absolute constant $C$. Since, for another absolute constant $C, \gamma(E) \leq C M^{1}(E)$ for all $\sigma$-compact $E$, we deduce that for each $z \in \partial_{e} X$ there exists $\delta_{z}>0$ such that

$$
M^{1}(\Delta(z, \delta) \backslash X) \geq C \delta, \quad 0<\delta<\delta_{z} .
$$

Now, arguing as in $\S \S 4$ and 5 in Chapter II of [19] we can prove that each $f \in$ $\mathrm{VMO}_{a}(X)$ can be approximated in $\operatorname{BMO}(X)$ be a sequence of functions $\left(f_{n}\right)$ in $\operatorname{VMO}(\mathbf{C})$ such that each $f_{n}$ is holomorphic on $\stackrel{\circ}{X}$ and on a neighborhood (depending on $n$ ) of $\partial_{e} X$. Since $\partial_{i} X$ is a $G_{\delta}$-set, we can apply 5.1 to each $f_{n}$ to get approximating functions which are holomorphic on a neighborhood of $X$.

6. BMO approximation of Lipschitz functions. Let $E$ be a subset of $\mathbf{C}$. For a function $f: E \rightarrow \mathbf{C}$ and for $0<\alpha<1$ set

$$
\|f\|_{\alpha, E}=\sup \frac{|f(x)-f(y)|}{|x-y|^{\alpha}}
$$

where the supremum is taken over all pairs $x, y \in E, x \neq y$. Write $\operatorname{Lip}(\alpha, E)$ for the space of those functions $f$ such that $\|f\|_{\alpha, E}<\infty$.

THEOREM. Let $X \subset \mathbf{C}$ be compact and suppose $0<\alpha<1$. Then the following are equivalent.

(i) Each function $f \in \operatorname{Lip}(\alpha, X)$ which is holomorphic on $\stackrel{\circ}{X}$ is the limit in $\mathrm{BMO}(X)$ of a sequence of rational functions with poles off $X$.

(ii) There exists a constant $C>0$ such that

$$
\left(M^{1+\alpha}(\Delta \backslash \stackrel{\circ}{X})\right)^{1 / 1+\alpha} \leq C M^{1}(\Delta \backslash X)
$$

for each open disc $\Delta$. 
(iii) There exists a constant $C>0$ such that

$$
\limsup _{\delta \rightarrow 0} \frac{M^{1}(\Delta(z, \delta) \backslash X)}{\delta} \geq C
$$

for $M^{1+\alpha}$ almost all $z \in \partial X$.

See [11 and 14] for previous results of this type. Before going on the proof let us recall the notion of $\alpha$-capacity. Given a compact $K \subset \mathbf{C}$ one defines

$$
\gamma_{\alpha}(K)=\sup \left\{\left|f^{\prime}(\infty)\right|: f \in A_{\alpha}(K)\right\}
$$

where $A_{\alpha}(K)$ is the set of those functions in $\operatorname{Lip}(\alpha, \mathbf{C})$ which are holomorphic on $K^{c}$ and satisfy $\|f\|_{\alpha, \mathbf{C}} \leq 1$. If $E$ is an arbitrary subset of $\mathbf{C}$ one writes

$$
\gamma_{\alpha}(E)=\sup \left\{\gamma_{\alpha}(K): K \text { compact } \subset E\right\} .
$$

A theorem of Melnikov [11] states that there is a constant $C=C(\alpha)>0$ such that

$$
C^{-1} M^{1+\alpha}(E) \leq \gamma_{\alpha}(E) \leq C M^{1+\alpha}(E)
$$

for each Borel subset $E$ of $\mathbf{C}$.

PROOF OF THEOREM 4. (i) $\Rightarrow$ (ii). Given an open disc $\Delta$ and $\varepsilon>0$ choose $f \in \operatorname{Lip}(\alpha, \mathbf{C})$ such that $f$ is holomorphic outside a compact subset of $\Delta \backslash \dot{X}$, $\|f\|_{\alpha, \mathbf{C}} \leq 1, f(\infty)=0$ and $\left|f^{\prime}(\infty)\right|>\gamma_{\alpha}(\Delta \backslash \stackrel{\circ}{X})-\varepsilon$. By hypothesis, there is a sequence $\left(f_{n}\right)$ of functions in $C_{0}^{\infty}(\mathbf{C})$, such that each $f_{n}$ is holomorphic in a neighborhood of $X$ and $f_{n_{\mid X}} \rightarrow f_{\mid X}$ in $\operatorname{BMO}(X)$. The modification argument used in the proof of (i) $\Rightarrow$ (ii) in Theorem 1 provides a sequence $\left(h_{n}\right)$ of functions, such that each $h_{n}$ is holomorphic outside a compact subset of $\Delta \backslash X$ and $h_{n} \rightarrow f$ in $\operatorname{BMO}(\mathbf{C})$. From $\left|h_{n}^{\prime}(\infty)\right| \leq\left\|h_{n}\right\|_{*} \gamma_{*}(\Delta \backslash X)$ we deduce, letting $n \rightarrow \infty$,

$$
\gamma_{\alpha}(\Delta \backslash \stackrel{\circ}{X})-\varepsilon \leq\left|f^{\prime}(\infty)\right| \leq\|f\|_{*} \gamma_{*}(\Delta \backslash X) \leq 2\|f\|_{\infty} \gamma_{*}(\Delta \backslash X) .
$$

By the estimate of the uniform norm in terms of the $\operatorname{Lip} \alpha$ norm $[\mathbf{1 4}, 7.6$, p. 110] we have

$$
\|f\|_{\infty} \leq C \gamma_{\alpha}(\Delta \backslash \stackrel{\circ}{X})^{\alpha / 1+\alpha}
$$

which, inserted in (14), gives

$$
\gamma_{\alpha}(\Delta \backslash \stackrel{\circ}{X})-\varepsilon \leq C \gamma_{\alpha}(\Delta \backslash \stackrel{\circ}{X})^{\alpha / 1+\alpha} \gamma_{*}(\Delta \backslash X) .
$$

To obtain (ii) simply let $\varepsilon \rightarrow 0$ in the last inequality and recall that $\gamma_{\alpha}$ and $M^{1+\alpha}$ are equivalent on Borel sets.

(ii) $\Rightarrow$ (iii). From (ii) we deduce, for each $z \in \partial X$,

$$
\begin{aligned}
\limsup _{\delta \rightarrow 0} M^{1}(\Delta(z, \delta) \backslash X) \delta^{-1} & \geq C \limsup _{\delta \rightarrow 0}\left(M^{1+\alpha}(\Delta(z, \delta) \backslash \dot{X}) \delta^{-(1+\alpha)}\right)^{1 / 1+\alpha} \\
& \geq C\left(\limsup _{\delta \rightarrow 0} M^{1+\alpha}(\Delta(z, \delta) \cap \partial X) \delta^{-(1+\alpha)}\right)^{1 / 1+\alpha} .
\end{aligned}
$$

By a well-known metric density lemma $[4,4.4$, p. 121] there exists an absolute constant $A>0$ such that

$$
\limsup _{\delta \rightarrow 0} M^{1+\alpha}(\Delta(z, \delta) \cap \partial X) \delta^{-(1+\alpha)} \geq A
$$

for $M^{1+\alpha}$ almost all $z \in \partial X$. This shows (iii). 
(iii) $\Rightarrow$ (i). We will follow closely Melnikov [11]. Let $f \in \operatorname{Lip}(\alpha, X)$ be holomorphic on $X$. Consider an extension of $f$ to a function in $\operatorname{Lip}(\alpha, \mathbf{C})$ and denote it again by $f$. If $\Delta$ is an open disc of radius $\delta$ and $\phi \in C_{0}^{1}(\Delta)$, then

$$
\begin{aligned}
\left|\int \bar{\partial} \phi(\xi) f(\xi) d m(\xi)\right| & =\left|\left(T_{\phi} f\right)^{\prime}(\infty)\right| \\
& \leq\left\|T_{\phi} f\right\|_{\alpha} \gamma_{\alpha}(\Delta \backslash \stackrel{\circ}{X}) \leq C \delta\|\nabla \phi\|_{\infty}\|f\|_{\alpha} \gamma_{\alpha}(\Delta \backslash \stackrel{\circ}{X})
\end{aligned}
$$

where we used, in the last inequality, the fact that $T_{\phi} \operatorname{maps} \operatorname{Lip}(\alpha, \mathbf{C}) \operatorname{into} \operatorname{Lip}(\alpha, \mathbf{C})$ continuously $[\mathbf{1 4}$, p. 105]. We are going to prove the inequality

$$
M^{1+\alpha}(\Delta \backslash \stackrel{\circ}{X}) \leq C \delta^{\alpha} M^{1}(\Delta \backslash X) .
$$

This, combined with Theorem 1 and (13), will give (i).

We clearly have

$$
M^{1+\alpha}(\Delta \backslash \stackrel{\circ}{X}) \leq M^{1+\alpha}(\Delta \backslash X)+M^{1+\alpha}(\Delta \cap \partial X)
$$

and

$$
\begin{aligned}
M^{1+\alpha}(\Delta \backslash X) & =\left(M^{1+\alpha}(\Delta \backslash X)\right)^{\alpha / 1+\alpha}\left(M^{1+\alpha}(\Delta \backslash X)\right)^{1 / 1+\alpha} \\
& \leq \delta^{\alpha} M^{1}(\Delta \backslash X) .
\end{aligned}
$$

We must now estimate $M^{1+\alpha}(\Delta \cap \partial X)$. Let $E$ be the set of points in $\Delta \cap \partial X$ which satisfy

$$
\limsup _{\delta \rightarrow 0} M^{1}(\Delta(z, \delta) \backslash X) \delta^{-1} \geq C
$$

where $C$ is the constant in condition (iii). Using (16) we can find discs $\Delta\left(z_{j}, \delta_{j}\right)$, with $z_{j} \in E$, satisfying $E \subset \bigcup_{j} \Delta\left(z_{j}, \delta_{j}\right), \Delta\left(z_{j}, \delta_{j}\right) \subset \Delta$ and

$$
M^{1}\left(\Delta\left(z_{j}, \delta_{j}\right) \backslash X\right) \geq C \delta_{j}, \quad \text { for all } j .
$$

The covering lemma in $\left[4\right.$, p. 122] provides a subsequence $\left(\Delta\left(z_{j_{n}}, \delta_{j_{n}}\right)\right)$ with the following properties:

(a) $\Delta\left(z_{j_{n}}, 2 \delta_{j_{n}}\right) \cap \Delta\left(z_{j_{m}}, 2 \delta_{j_{m}}\right)=\varnothing, m \neq n$.

(b) $\sum_{\Delta_{j_{n}} C D} \delta_{j_{n}} \leq 2 \rho$ for each disc $D$ of radius $\rho$.

(c) $\sum_{n} \delta_{j_{n}} \geq C M^{1}\left(\bigcup_{j} \Delta\left(z_{j}, \delta_{j}\right)\right)$.

Thus

$$
\begin{aligned}
\left(M^{1+\alpha}(\Delta \cap \partial X)\right)^{1 / 1+\alpha} & \leq\left(M^{1+\alpha}(E)\right)^{1 / 1+\alpha} \leq M^{1+\alpha}\left(\bigcup_{j} \Delta\left(z_{j}, \delta_{j}\right)\right)^{1 / 1+\alpha} \\
& \leq M^{1}\left(\bigcup_{j} \Delta\left(z_{j}, \delta_{j}\right)\right) \leq C \sum_{n} \delta_{j_{n}} .
\end{aligned}
$$

From the Frostman Lemma $[4,1.5$, p. 62] and (17) we get, for each $n$, a positive measure $\mu_{n}$ supported on a compact subset of $\Delta_{j_{n}} \backslash X$, with $\left\|\mu_{n}\right\| \geq C \delta_{j_{n}}$ and $\mu_{n}(\Delta(z, \delta)) \leq \delta, z \in \mathbf{C}, \delta>0$. Set $\mu=\sum_{n} \mu_{n}$. It is sufficient to show that

$$
\|\hat{\mu}\|_{*} \leq C
$$


for some absolute constant $C$, because then

$$
\begin{aligned}
\left(M^{1+\alpha}(\Delta \cap \partial X)\right)^{1 / 1+\alpha} & \leq C \sum_{n} \delta_{j_{n}} \leq C \sum_{n}\left\|\mu_{n}\right\| \\
& =C(\hat{\mu})^{\prime}(\infty) \leq C \gamma_{*}(\Delta \backslash X),
\end{aligned}
$$

and hence

$$
M^{1+\alpha}(\Delta \cap \partial X) \leq C M^{1}(\Delta \backslash X)^{1+\alpha} \leq C \delta^{\alpha} M^{1}(\Delta \backslash X),
$$

which completes the proof of (15).

To show (18) it is enough to prove, according to (4), that $\mu(D) \leq 10 \rho$ for each disc $D$ of radius $\rho$. Fix a such disc $D$, and consider two cases.

Case 1. There exists $n$ with $D \cap \Delta_{j_{n}} \neq \varnothing$ and $\delta_{j_{n}} \geq 2 \rho$. Then $D \subset 2 \Delta_{j_{n}}$, and so $D \cap \Delta_{j_{m}}=\varnothing$ for $m \neq n$, by (a). Thus

$$
\mu(D)=\sum_{m} \mu_{m}(D)=\mu_{n}(D) \leq \rho
$$

Case 2. For each $n, \delta_{j_{n}}<2 \rho$ whenever $D \cap \Delta_{j_{n}} \neq \varnothing$. Then $\Delta_{j_{n}} \subset 5 D$ whenever $D \cap \Delta_{j_{n}} \neq \varnothing$. Therefore

$$
\begin{aligned}
\mu(D) & =\sum_{D \cap \Delta_{j_{n}} \neq \varnothing} \mu_{n}(D) \leq \sum_{\Delta_{j_{n} \subset 5 D}} \mu_{n}(D) \\
& \leq \sum_{\Delta_{j_{n}} \subset 5 D} \mu_{n}\left(\Delta_{j_{n}}\right) \leq \sum_{\Delta_{j_{n}} \subset 5 D} \delta_{j_{n}} \leq 10 \rho,
\end{aligned}
$$

where in the last inequality we applied (b).

7. Examples.

1. Classical Swiss cheese. A Swiss cheese is a compact obtained by deleting from the closed unit disc a sequence of open nonintersecting discs such that the sum of their radii is finite. It turns out that if $X$ is a Swiss cheese then $\operatorname{VMO}_{a}(X) \subsetneq R_{*}(X)$. This follows from the argument in $[12$, p. 205] for $\alpha=0$.

2. We are going to construct a compact $X$ without interior, such that $R(X) \neq$ $C(X)$ but $C(X) \subset R_{*}(X)$. The starting point for our construction is the planar Cantor set $E$ obtained from the unit square $Q=I \times I, I=[0,1]$, by taking the "corner quarters." See $[6$, or 4, p. 87]. This set has the property that $\gamma(E)=0$ but $M^{1}(E)=m>0$. Given any square $S$ with sides parallel to the coordinate axis, consider a dilation $d$ and a translation $t$ so that $S=(t \circ d)(Q)$. We will call $(t \circ d)(E)$ "the Cantor set constructed inside $S$ ".

Step 1. Subdivide $Q$ in 16 squares of side $\frac{1}{4}$. Let $Q_{j}^{(1)}, 1 \leq j \leq 4$, be the four corner squares, and let $R_{j}^{(1)}$ be the remaining 12 squares. Define $K_{1}=\bigcup_{j} Q_{j}^{(1)}$. Repeat the process with each $Q_{j}^{(1)}$ in place of $Q$. Let $Q_{j}^{(2)}$ be the 16 corner squares of side $\frac{1}{16}$ obtained inside the $Q_{j}^{(1)}$ 's and let $R_{j}^{(2)}$ be the remaining 48 squares lying inside the $Q_{j}^{(1)}$ 's. Set $K_{2}=\bigcup_{j} Q_{j}^{(2)}$. In this way we construct inductively families of 4-adic squares $\left(Q_{j}^{(n)}\right)_{j}$ and $\left(R_{j}^{(n)}\right)_{j}$, and sets $K_{n}=\bigcup_{j} Q_{j}^{(n)}$. Since $E=\bigcap K_{n}$, $\gamma\left(K_{n}\right) \rightarrow 0$ as $n \rightarrow \infty$. Fix $\gamma, 0<\gamma<\frac{1}{2}$, and choose $\nu$ so that $\gamma\left(K_{\nu}\right)<\gamma$. Define $U_{1}=\lambda_{1} \stackrel{\circ}{K}_{\nu}$, where $\lambda_{1}>1$ is sufficiently close to 1 so that $\gamma\left(\bar{U}_{1}\right)<\gamma$. 
Step 2. We repeat the construction of Step 1 inside each $R_{j}^{(k)}, 1 \leq k \leq \nu$, obtaining at the $n$th step families of squares $\left(Q_{j}^{(k, n)}\right)_{j, k}$ and $\left(R_{j}^{(k, n)}\right)_{j, k}$, and sets $H_{n}=\bigcup_{j, k} Q_{j}^{(k, n)}$. If $E_{j, k}$ is the Cantor set constructed inside $R_{j}^{(k)}$, then

$$
\bigcap_{n}\left(\bar{U}_{1} \cup H_{n}\right)=\bar{U}_{1} \cup\left(\bigcup_{j, k} E_{j, k}\right) .
$$

It is well known (and quite easy to show) that if $K_{1}, K_{2}$ are compact and $\gamma\left(K_{2}\right)=0$, then $\gamma\left(K_{1} \cup K_{2}\right)=\gamma\left(K_{1}\right)$. Therefore, since $\gamma\left(E_{j, k}\right)=0$ for all $j$ and $k$,

$$
\gamma\left(\bar{U}_{1} \cup\left(\bigcup_{j, k} E_{j, k}\right)\right)=\gamma\left(\bar{U}_{1}\right),
$$

and thus $\gamma\left(\bar{U}_{1} \cup H_{n}\right) \rightarrow \gamma\left(\bar{U}_{1}\right)$ as $n \rightarrow \infty$. Therefore, if $\nu$ is large enough, $\gamma\left(\bar{U}_{1} \cup H_{\nu}\right)<\gamma$. Set $U_{2}=\lambda_{2} \stackrel{\circ}{H}_{\nu}$, where $\lambda_{2}>1$ is sufficiently close to 1 so that $\gamma\left(\bar{U}_{1} \cup \bar{U}_{2}\right)<\gamma$.

Step 3 . We construct inductively a sequence of open sets $\left(U_{n}\right)$ such that

$$
\gamma\left(\bar{U}_{1} \cup \bar{U}_{2} \cup \cdots \cup \bar{U}_{n}\right)<\gamma, \quad n=1,2, \ldots
$$

Write $X=Q \backslash\left(\bigcup_{n=1}^{\infty} U_{n}\right)$. Let us prove that $R(X) \neq C(X)$. Denote by $\Delta$ the open disc with center $\frac{1}{2}+i\left(\frac{1}{2}\right)$ and radius $\frac{1}{2}$. Since $\Delta \backslash X \subset \bigcup_{n=1}^{\infty} U_{n}, \gamma(\Delta \backslash X) \leq$ $\gamma\left(\bigcup_{n=1}^{\infty} U_{n}\right)$. Now, each compact subset of $\bigcup_{n=1}^{\infty} U_{n}$ is contained in a finite union of $U_{n}$ 's, so that, according to (19), $\gamma\left(\bigcup_{n=1}^{\infty} U_{n}\right) \leq \gamma$ and thus $\gamma(\Delta \backslash X)<\frac{1}{2}$. Therefore $R(X) \neq C(X)$.

In view of the corollary to Theorem 2 , the inclusion $C(X) \subset R_{*}(X)$ will follow if we show that $M^{1}(2 \Delta \backslash X) \geq m \delta / 4$ for each disc $\Delta$ of radius $\delta \leq 1$ with center in $Q$. If $\Delta$ is such a disc and $n$ is a nonnegative integer such that $4^{-(n+1)}<\delta \leq 4^{-n}$, then $2 \Delta$ contains a 4 -adic square $Q^{*}$ of side length $4^{-(n+1)}$ contained in $Q$. According to the way $X$ has been defined, $Q^{*} \cap X^{c}$ contains the Cantor set $E^{*}$ constructed inside $Q^{*}$. Therefore

$$
M^{1}(2 \Delta \backslash X) \geq M^{1}\left(Q^{*} \backslash X\right) \geq M^{1}\left(E^{*}\right)=4^{-(n+1)} m \geq m \delta / 4 .
$$

3. We construct a family of examples showing that the condition on the inner boundary in 5.2 is sharp.

Given a nowhere dense compact $E$ with $M_{*}^{1}(E)>0$ (i.e., $E$ does not have $\sigma$-finite length) there exists a compact $X$ such that $\partial_{i} X=E$ and $\mathrm{VMO}_{a}(X) \not \subset R_{*}(X)$.

PROOF. According to Theorem 2, a necessary condition for $\mathrm{VMO}_{a}(X) \subset R_{*}(X)$ is that

$$
M_{*}^{1}(\Delta \backslash \stackrel{\circ}{X}) \leq C M^{1}(\Delta \backslash X)
$$

for all open discs $\Delta$ and for some numerical constant $C>0$.

Let $\Delta$ be an open disc containing $E$ and let $\left(\Delta_{n}\right)$ be a sequence of open discs with radii $r_{n}$ such that

(i) $\bar{\Delta}_{n} \subset \Delta \backslash E$, for all $n$.

(ii) $\bar{\Delta}_{n} \cap \bar{\Delta}_{m}=\varnothing$, whenever $n \neq m$.

(iii) The accumulation points of $\bigcup_{n} \Delta_{n}$ are exactly the points in $E$.

(iv) $\sum r_{n}<(2 C)^{-1} M_{*}^{1}(E)$. 
Define $X=\bar{\Delta} \backslash \bigcup_{n} \Delta_{n}$, so that $\partial_{i} X=E$ and $\Delta \backslash X=\bigcup_{n} \Delta_{n}$. We have

$$
M^{1}(\Delta \backslash X) \leq \sum 2 r_{n}<C^{-1} M_{*}^{1}(E) \leq C^{-1} M_{*}^{1}(\Delta \backslash \stackrel{\circ}{X}) .
$$

Thus condition (20) is violated and $X$ has the desired properties.

4. We construct now a compact $X$ for which uniform rational approximation holds but BMO rational approximation does not hold, i.e., for which $R(X)=A(X)$ and $\mathrm{VMO}_{a}(X) \not \subset R_{*}(X)$.

Let $E$ be the planar Cantor set associated to the sequence $\lambda_{n}=\frac{1}{4}(1+1 /(n+1))$, $n=1,2, \ldots$. Recall (see $\left[4\right.$, p. 87]) that at the $n$th step one considers $4^{n}$ closed squares $Q_{n, j}, 1 \leq j \leq 4^{n}$ of side length $\sigma_{n}=\lambda_{1} \lambda_{2} \cdots \lambda_{n}$. Then one sets $E_{n}=$ $\bigcup_{j} Q_{n, j}$ and $E=\bigcap_{n} E_{n}$. Because of the particular choice of $\left(\lambda_{n}\right)$ we have $\gamma(E)=0$ and $M_{*}^{1}(E)>0$. Take now open discs $r_{n, j}$ with the same center as $Q_{n, j}$ and radii $r_{n, j}$, satisfying

(i) $\sum r_{n, j}<(2 C)^{-1} M_{*}^{1}(E), C$ being the constant in the previous example.

(ii) $\bar{\Delta}_{n, j}$ does not intersect $E_{n+1}$.

Let $\Delta$ be an open disc containing $E$, and define $X=\bar{\Delta} \backslash \bigcup_{n, j} \Delta_{n, j}$. We have $\mathrm{VMO}_{a}(X) \not \subset R_{*}(X)$ because of Example 3. We will choose the $\Delta_{n, j}$ so that in addition $R(X)=A(X)$.

We claim that to get $R(X)=A(X)$ is enough that

$$
\alpha\left(Q_{n, j} \backslash \stackrel{\circ}{X}\right) \leq C \alpha\left(Q_{n, j} \backslash X\right), \quad \text { for all } n \text { and } j .
$$

To prove the claim let us show that (21) implies

$$
\alpha(\Delta(a, \delta) \backslash \stackrel{\circ}{X}) \leq C \alpha(\Delta(a, 8 \delta) \backslash X), \quad a \in E,
$$

for sufficiently small $\delta>0$.

Given $a \in E$ and $\delta>0$ choose $n, j$ and $k$ so that $\sigma_{n+1} \leq \delta<\sigma_{n}$ and $a \in$ $Q_{n+1, k} \subset Q_{n, j}$.

If $\delta$ is small enough we have $\Delta(a, \delta) \backslash \stackrel{\circ}{X} \subset Q_{n, j} \backslash \stackrel{\circ}{X}$. On the other hand $Q_{n, j} \subset \Delta(a, 8 \delta)$ because $\operatorname{diam} Q_{n, j}=\sqrt{2} \sigma_{n}<8 \delta$.

Therefore

$$
\alpha(\Delta(a, \delta) \backslash \stackrel{\circ}{X}) \leq \alpha\left(Q_{n, j} \backslash \stackrel{\circ}{X}\right) \leq C \alpha\left(Q_{n, j} \backslash X\right) \leq C \alpha(\Delta(a, 8 \delta) \backslash X) .
$$

To get (21) we first consider open discs $D_{n, j}$ with the same center as $Q_{n, j}$ and radii $\rho_{n, j}$ satisfying (i) and (ii) with $r_{n, j}$ replaced by $\rho_{n, j}$ and $\Delta_{n, j}$ by $D_{n, j}$. We will define inductively the discs $\Delta_{n, j}$ so that

$$
\gamma\left(E \cup\left(\bigcup_{m \geq n+1} \bigcup_{k} \bar{\Delta}_{m, k}\right)\right) \leq \alpha\left(\Delta_{n, j}\right)
$$

for $n=0,1, \ldots$, and $1 \leq j \leq 4^{n}$.

We set $\Delta_{0,1}=D_{0,1}$ and proceed to define $\Delta_{1, j}, 1 \leq j \leq 4$. If $z_{n, j}$ is the center of $Q_{n, j}$ then

$$
\bigcap_{\varepsilon>0}\left(E \cup\left(\bigcup_{n \geq 1} \bigcup_{j} \varepsilon \bar{D}_{n, j}\right)\right)=E \cup\left\{z_{n, j} / n \geq 1, j\right\} .
$$


Since the set in the right side has zero analytic capacity, we can find $\varepsilon$ such that $0<\varepsilon<1$ and

$$
\gamma\left(E \cup\left(\bigcup_{n \geq 1} \bigcup_{j} \varepsilon \bar{D}_{n, j}\right)\right)<\alpha\left(\Delta_{0,1}\right) .
$$

Set $\Delta_{1, j}=\varepsilon D_{1, j}, 1 \leq j \leq 4$.

An obvious inductive argument completes now the construction of the sequence $\left(\Delta_{n, j}\right)$.

Appealing to Melnikov's estimate [3, p. 234] we get

$$
\begin{aligned}
\alpha\left(Q_{n, j} \backslash \stackrel{\circ}{X}\right) & \leq C\left(\alpha\left(Q_{n, j} \backslash\left(\stackrel{\circ}{X} \cup \bar{\Delta}_{n, j}\right)\right)+\alpha\left(\bar{\Delta}_{n, j}\right)\right) \\
& \leq C\left(\alpha\left(E \cup\left(\bigcup_{m \geq n+1} \bigcup_{k} \bar{\Delta}_{m, k}\right)\right)+\alpha\left(\Delta_{n, j}\right)\right) \\
& \leq C \alpha\left(\Delta_{n, j}\right) \leq C \alpha\left(Q_{n, j} \backslash X\right)
\end{aligned}
$$

which is (21).

ACKNOWLEDGMENTS. I would like to express my gratitude to the Department of Mathematics of UCLA for its sunny hospitality during the academic year 198384. In particular, I am indebted to T. W. Gamelin and to J. B. Garnett for many interesting conversations on the subject. Thanks are also due to A. G. O'Farrell for some valuable correspondence.

\section{REFERENCES}

1. L. Carleson, Selected problems on exceptional sets, Van Nostrand, Princeton, N. J., 1967.

2. A. M. Davie, Analytic capacity and approximation problems, Trans. Amer. Math. Soc. 171 (1972), 409-444.

3. T. W. Gamelin, Uniform algebras, Prentice-Hall, Englewood Cliffs, N. J., 1969.

4. J. B. Garnett, Analytic capacity and measure, Lecture Notes in Math., vol. 297, SpringerVerlag, Berlin and New York, 1972.

5. __ Bounded analytic functions, Academic Press, London and New York, 1981.

6. $\ldots$, Positive length but zero analytic capacity, Proc. Amer. Math. Soc. 24 (1970), 696699.

7. _ Metric conditions for rational approximation, Duke Math. J. 37 (1970), 689-699.

8. R. Harvey and J. Polking, Removable singularities of solutions of linear partial differential equations, Acta Math. 125 (1970), 39-56.

9. R. Kaufman, Hausdorff measure, BMO, and analytic functions, Pacific J. Math. 102 (1982), 369-371.

10. P. Lindberg, $A$ constructive method for $L^{p}$-approximation by analytic functions, Ark. Math. 20 (1982), 61-68.

11. M. S. Melnikov, Metric properties of analytic $\alpha$-capacity and approximation of analytic functions with Holder condition by rational functions, Math. USSR-Sb. 8 (1969), 115-124.

12. M. S. Melnikov and A. G. Vitushkin, Analytic capacity and rational approximation, Lecture Notes in Math., vol. 1043, Springer-Verlag, Berlin and New York, 1983, pp. 495-497.

13. A. G. O'Farrell, Hausdorff content and rational approximation in fractional Lipschitz norms, Trans. Amer. Math. Soc. 228 (1977), 187-206.

14. _ Estimates for capacities and approximation in Lipschitz norms, J. Reine Angew Math. 311/312 (1979), 101-115.

15. __ Qualitative rational approximation on plane compacta, Lecture Notes in Math., vol. 995, Springer-Verlag, Berlin and New York, 1983, pp. 103-122.

16. C. Sadosky, Interpolation of operators and singular integrals, Dekker, New York and Basel, 1979. 
17. D. Sarason, Functions of vanishing mean oscillation, Trans. Amer. Math. Soc. 207 (1975), 391-405.

18. S. Spanne, Some function spaces defined using the mean oscillation over cubes, Ann. Scuola Norm. Sup. Pisa Cl. Sci. 19 (1965), 593-608.

19. A. G. Vitushkin, Analytic capacity of sets in problems of approximation theory, Russian Math. Surveys 22 (1967), 139-200.

20. T. H. Wolff, Restrictions of $A_{p}$ weights, preprint.

Department of Mathematics, University of California, los Angeles, CaliFORNIA 90024

Current address: Secció de Matemàtiques, Universitat Autònoma de Barcelona, Bellaterra (Barcelona), Spain 